\title{
Poor Outcomes in Head and Neck Non-Melanoma Cutaneous Carcinomas ${ }^{\S}$
}

\author{
Kevin C. Huoh and Steven J. Wang* \\ Head and Neck Surgery and Oncology, UCSF Helen Diller Family Comprehensive Cancer Center, University of \\ California, San Francisco, San Francisco, CA, USA
}

\begin{abstract}
Objectives: Head and neck nonmelanoma cutaneous carcinomas (NMCC) may have poor outcomes, such as metastasis and recurrence. It remains unclear which patients are at increased risk for poor outcomes. We characterize a population of NMCC seen in a head and neck surgical practice. We sought to identify predictors of poor outcome in head and neck NMCC.

Study Design: A retrospective cohort study of 161 patients with head and neck NMCC who presented to a university based otolaryngology-head and neck surgery practice over a 13 year period was performed. The study group included 127 men and 34 women, age range: 33 - 92 years.

Subjects and Methods: Tumor characteristics of the study population were recorded, and statistical analysis and KaplanMeier survival calculations were performed to determine predictors of poor outcomes.

Results: 113 squamous cell carcinomas (SCC) and 48 basal cell carcinomas (BCC) were identified. Among SCC patients, there was a $44 \%$ incidence of metastasis and a $47 \%$ incidence of recurrence. Among BCC patients, there was a $10 \%$ incidence of metastasis and a 50\% incidence of recurrence. Twenty percent of SCC patients had a history of immunosuppression. Among SCC patients with immunosuppression, 83\% had metastasis or recurrence. Survival for SCC patients with metastatic disease was significantly worse than patients without metastases. The presence of parotid metastases in SCC conferred a survival advantage when compared to other sites of metastases.
\end{abstract}

Conclusion: The incidence of poor outcomes in NMCC was higher in our series than reported in the literature. Survival patterns of our study parallel those of similar studies in the literature.

Keywords: Cutaneous squamous cell carcinoma, nonmelanoma skin cancer, basal cell carcinoma, metastasis, recurrence.

\section{INTRODUCTION}

Nonmelanoma cutaneous carcinomas (NMCC) comprise the most common malignancies in the United States. Approximately 1.3 million cases are treated annually, and the incidence continues to rise [1]. Basal cell carcinomas (BCC) and squamous cell carcinomas (SCC) comprise the majority of NMCC. The most common location for these malignancies is in the head and neck region which is in accordance with the major known risk factor of chronic sun exposure [2].

The majority of skin cancers are diagnosed by primary care physicians and dermatologists who are able to successfully treat these lesions by simple, local extirpation which is curative in most cases [3]. However, some patients with more complex locally metastatic or recurrent disease may be seen by otolaryngologists- head and neck surgeons for NMCC of the head and neck.

\footnotetext{
*Address correspondence to this author at the U UCSF Dept of Otolaryngology-Head and Neck Surgery, 2233 Post St, Box 1225, San Francisco, CA 94115, USA; Tel: (415) 885-7521; Fax: (415) 885-7546; E-mail: swang@ohns.ucsf.edu

${ }^{\S}$ This manuscript was presented at the Annual Meeting of the American Academy of Otolaryngology-Head and Neck Surgery Foundation, October
} 4-7, 2009.
Basal cell carcinomas comprise nearly $75 \%$ of cases of NMCC in the United States. Of these lesions, nearly $90 \%$ occur in the head and neck region [1]. While a relatively common etiology for primary skin cancer, BCC is unlikely to cause metastatic disease. The quoted incidence of BCC metastases in the literature ranges from $0.0028 \%$ to $0.5 \%$ [2]. Squamous cell carcinomas comprise $20 \%$ of NMCC. Similarly, most of these lesions occur in the head and neck region. Unlike BCC, SCC has a greater propensity to metastasize with rates in the literature ranging from 0.5 to $16 \%$ [4]. Because most patients with NMCC never progress to developing metastatic or recurrent disease, the presence of either can be considered a poor outcome.

Several recent reports in the literature have focused on identifying patient and disease factors that may be predictive of poor outcomes for NMCC. Histopathologic factors such as lymphovascular invasion, poorly differentiated histology, perineural invasion, depth of invasion, and lesion size have been associated with lymph node metastases and recurrence [1]. Patient factors such as a history of immunosuppression have also been identified as a potential risk factor for poor outcomes in patients with NMCC [5].

In this study, we analyze the NMCC population seen at our tertiary university based head and neck surgical practice. We analyze the survival and outcomes of patients in our 
cohort. We also attempt to identify risk factors leading to poor outcomes in NMCC.

\section{METHODS}

The study protocol was approved by the University of California, San Francisco (UCSF) Committee on Human Research. Patients were identified by using a cutaneous carcinoma diagnosis based ICD-9 code search of the UCSF Department of Otolaryngology- Head and Neck Surgery billing database. This search identified patients treated from 1996-2009. Patients with melanoma and Merkel cell carcinoma were excluded. Patients who were seen in the department for primarily reconstructive purposes were also excluded from the study.

A retrospective chart review was then performed through accessing the electronic medical record. Data on each patient was recorded in a computerized database (Microsoft Excel; Microsoft, Seattle, Washington). Basic demographic information was culled including gender, date of birth, and age of diagnosis. Any history of immunosuppression was also noted. Immunosuppression was defined as patients with a history of solid organ transplantation, history of hematologic malignancy, or patients taking immunosuppressive medications for autoimmune conditions.

Histopathological data including location of original lesion, size, depth, perineural invasion, and margin status were gathered. Further data including metastatic disease, time to metastases, location of metastases, and treatment of metastases were recorded. Similar information about recurrent disease was also tabulated.

Metastatic disease was defined as pathologically verified disease not involving the skin. Recurrent disease was defined as new lesions in the same location as the previously treated disease. "Poor outcomes" include the presence of either recurrent or metastatic disease.

In order to derive survival data on the patients, the United States Social Security Death Index was employed to determine the vital status of patients and the date of death for those deceased. Follow-up time was determined through the electronic medical record.

Basic descriptive statistics on the patients were performed using SPSS (SPSS, Chicago, Illinois.) Associations between groups and patient characteristics were calculated using Fisher's exact test through SPSS. The Kaplan-Meier method was then applied to analyze survival data. Comparison of Kaplan-Meier curves was performed using the log-rank test.

\section{RESULTS}

Over the study interval from 1996-2009, 48 patients with basal cell carcinoma and 113 patients with cutaneous squamous cell carcinoma were treated in the Department of Otolaryngology- Head and Neck Surgery at the University of California, San Francisco. The demographic features of these two groups are highlighted in Table 1. There was no statistically significant difference between the groups in terms of gender or age.

For both BCC and SCC, the ear was the most common site of primary disease. $20.8 \%$ of the $\mathrm{BCC}$ patients had primary lesions of the ear and $22.1 \%$ of SCC patients had primary lesions of the ear. For BCC, the next most common sites were the cheek and nose. For SCC, the cheek and external ear canal were the next most common sites of disease.

Table 1. Patient Demographics

\begin{tabular}{|l||c|c|}
\hline & Basal Cell Cancer & Squamous Cell Cancer \\
\hline \hline Total Patients (n) & 48 & 113 \\
\hline Gender (ratio M:F) & $\begin{array}{c}37 \text { men } \\
(3.4: 1)\end{array}$ & $\begin{array}{c}90 \mathrm{men} \\
23 \text { women } \\
(4.1: 1)\end{array}$ \\
\hline $\begin{array}{l}\text { Average Age on } \\
\text { Presentation }\end{array}$ & $62.2+/-11.9$ years & $70.6+/-11.8$ years \\
\hline Age Range & $41-88$ years & $33-92$ years \\
\hline Median Age & 60 years & 70 years \\
\hline $\begin{array}{l}\text { Average } \\
\text { follow up time }\end{array}$ & 2.1 years & 1.2 years \\
\hline
\end{tabular}

In the basal cell carcinoma population, poor outcomes were seen in $54 \%$ of patients (Table 2). Metastatic disease occurred in $10 \%$ of the patients. Recurrence was present in $50 \%$ of patients. Six percent had both metastatic disease and disease recurrence. The cutaneous squamous cell carcinoma population demonstrated $70 \%$ with poor outcomes. Fortyfour percent had metastatic disease while $47 \%$ had recurrence. Both metastatic disease and recurrence occurred in $25 \%$.

Table 2. Poor Outcomes in Basal Cell Carcinomas and Squamous Cell Carcinomas

\begin{tabular}{|c|c|c|c|}
\hline & $\begin{array}{c}\text { Basal Cell } \\
(\mathbf{n}=\mathbf{4 8})\end{array}$ & $\begin{array}{c}\text { Squamous Cell } \\
(\mathbf{n}=\mathbf{1 1 3})\end{array}$ & $\boldsymbol{p}$ Value $^{\#}$ \\
\hline \hline Poor Outcomes & $26(54 \%)$ & $79(70 \%)$ & $0.07^{* *}$ \\
\hline Metastatic Disease & $5(10 \%)$ & $50(44 \%)$ & $0.0001^{*}$ \\
\hline Recurrence & $24(50 \%)$ & $53(47 \%)$ & 0.73 \\
\hline Both & $3(6 \%)$ & $28(25 \%)$ & -- \\
\hline
\end{tabular}

Fisher's exact test.

* Statistically significant $(\mathrm{p}<0.05)$

**Approaches statistical significance.

As expected, metastatic disease was significantly more likely in SCC compared with BCC $(\mathrm{p}<0.001)$. There was no significant difference between the two groups with respect to incidence of recurrent disease $(\mathrm{p}=0.73)$. The difference in poor outcomes trended towards statistical significance with an increased incidence of poor outcomes in the SCC group $(\mathrm{p}=0.07)$.

The locations of metastatic disease are shown in Table 3. Parotid and neck lymph nodes were the most common sites of metastatic disease respectively in both groups. The majority of SCC patients had disease isolated to the parotid region. Isolated cervical lymph node metastases were next most common sites of disease. A smaller number of patients had both parotid and cervical burden of disease. 
Table 3. Site of Metastatic Disease

\begin{tabular}{|c|c|c|}
\hline & Basal Cell $(\mathbf{n}=\mathbf{5})$ & Squamous Cell $(\mathbf{n}=\mathbf{5 0})$ \\
\hline \hline Parotid nodes & $2(40 \%)$ & $25(50 \%)$ \\
\hline Cervical nodes & $2(40 \%)$ & $15(30 \%)$ \\
\hline Both & $1(20 \%)$ & $8(16 \%)$ \\
\hline Distant metastases & -- & $2(4 \%)$ \\
\hline
\end{tabular}

Immunosuppressed patients with SCC (Table 4) had a higher rate of metastatic disease and poor outcomes than non-immunosuppressed patients; however, this difference was not statistically significant. The incidence of recurrence was higher in the non-immunosuppressed patients $(\mathrm{p}=0.03)$.

Table 4. Immunosuppression and Poor Outcomes in SCC

\begin{tabular}{|c|c|c|c|}
\hline & $\begin{array}{c}\text { Immunosuppressed } \\
(\mathbf{n}=\mathbf{2 3})\end{array}$ & $\begin{array}{c}\text { Non-Immunosuppressed } \\
(\mathbf{n}=\mathbf{9 0})\end{array}$ & $\boldsymbol{p}$ Value $^{\#}$ \\
\hline \hline $\begin{array}{c}\text { Poor } \\
\text { Outcomes }\end{array}$ & $19(83 \%)$ & $60(67 \%)$ & 0.20 \\
\hline $\begin{array}{c}\text { Metastatic } \\
\text { Disease }\end{array}$ & $13(57 \%)$ & $37(41 \%)$ & 0.24 \\
\hline Recurrence & $6(26 \%)$ & $47(52 \%)$ & $0.03 *$ \\
\hline
\end{tabular}

"Fisher's exact test.

*Statistically significant $(\mathrm{p}<0.05)$.

Kaplan-Meier survival analysis was carried out. BCC patients had a statistically significant higher survival than SCC patients $(\mathrm{p}<0.001)$ (Fig. 1). Subgroup survival analysis of the SCC population was performed. At two years, patients with poor outcomes exhibit lower survival than patients without poor outcomes (Fig. 2). However, with further follow-up, there was no statistically significant survival difference in patients without poor outcomes compared to those without $(\mathrm{p}=0.21)$. Metastatic disease alone resulted in

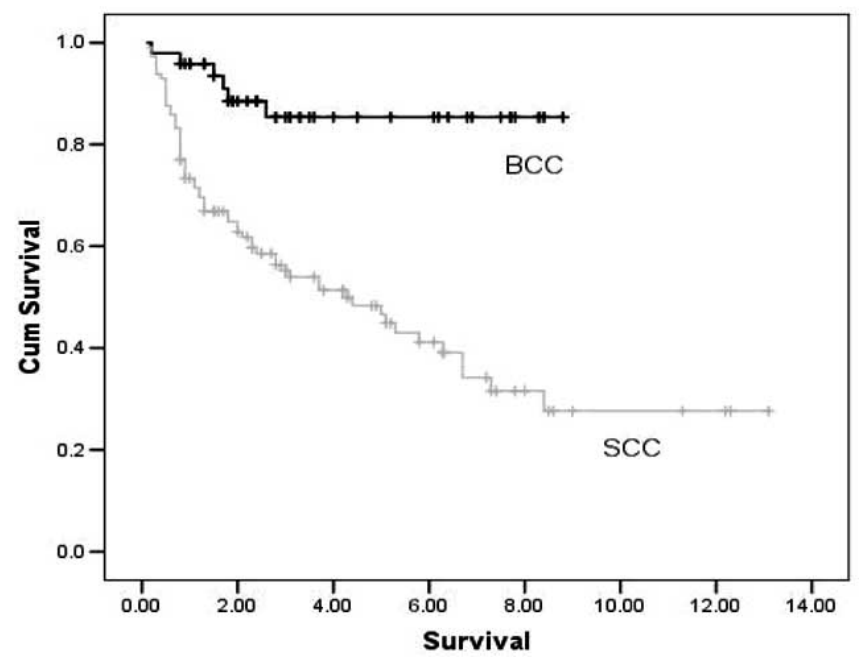

Fig. (1). Kaplan-Meier Survival Curves demonstrating differences among subgroups within the study. Graphs plot cumulative survival against survival in years. Basal Cell Carcinoma vs Squamous Cell Carcinoma, $\mathrm{p}<0.001 *$.

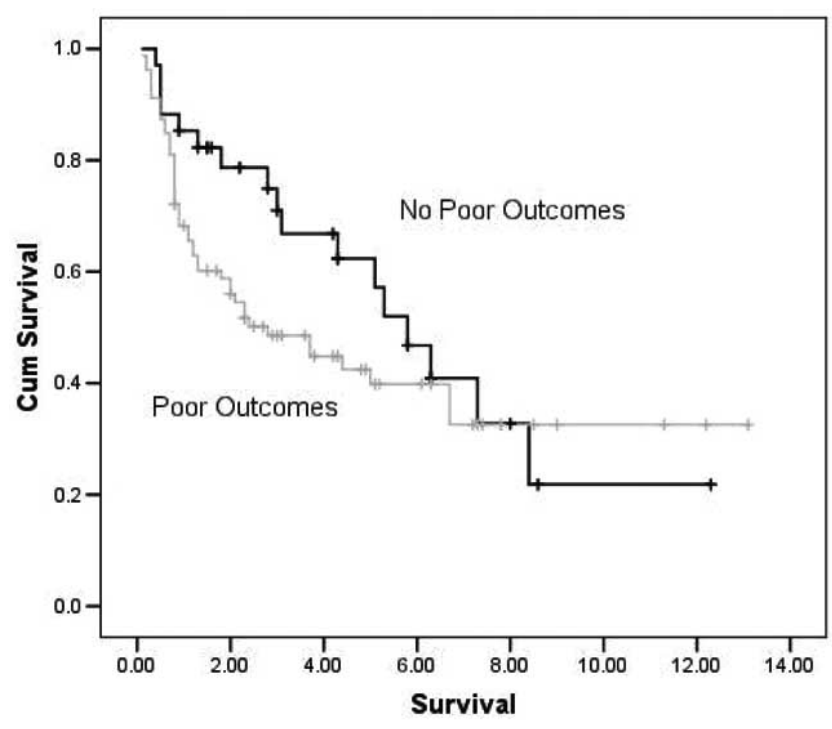

Fig. (2). Kaplan-Meier Survival Curves demonstrating differences among subgroups within the study. Graphs plot cumulative survival against survival in years. Poor Outcomes in SCC vs No Poor Outcomes in SCC, $\mathrm{p}=0.211$.

statistically significant poorer survival compared to those patients without metastatic disease ( $\mathrm{p}=0.02$ ) (Fig. 3). Recurrent disease, however, did not predict a statistically significant survival difference $(\mathrm{p}=0.80)$ (Fig. 4). Immunosuppressed patients with SCC fared worse, in terms of survival, particularly in the first 3 years (Fig. 5). However, with further follow-up, there was no statistically significant worse survival seen in immunosuppressed patients with SCC.

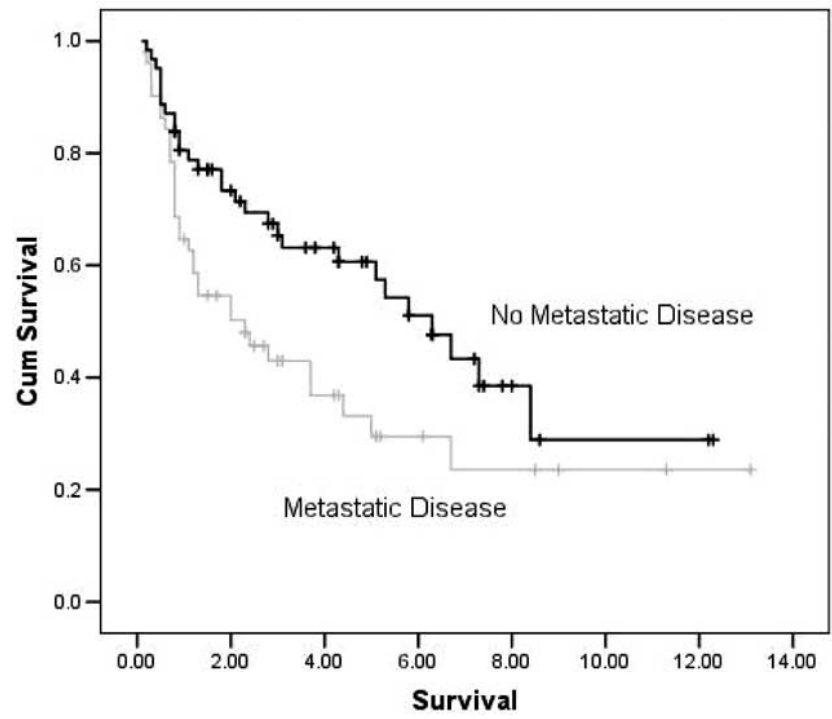

Fig. (3). Kaplan-Meier Survival Curves demonstrating differences among subgroups within the study. Graphs plot cumulative survival against survival in years. Metastatic Disease in SCC vs No Metastatic Disease in SCC, $\mathrm{p}=0.021$ *

Among SCC patients with metastases, there were notable survival differences dependent on the sites of metastatic disease. Survival of patients with isolated parotid metastases was better than patients with other sites of metastatic disease including parotid + neck and isolated neck disease $(\mathrm{p}=0.03)$ (Fig. 6). 


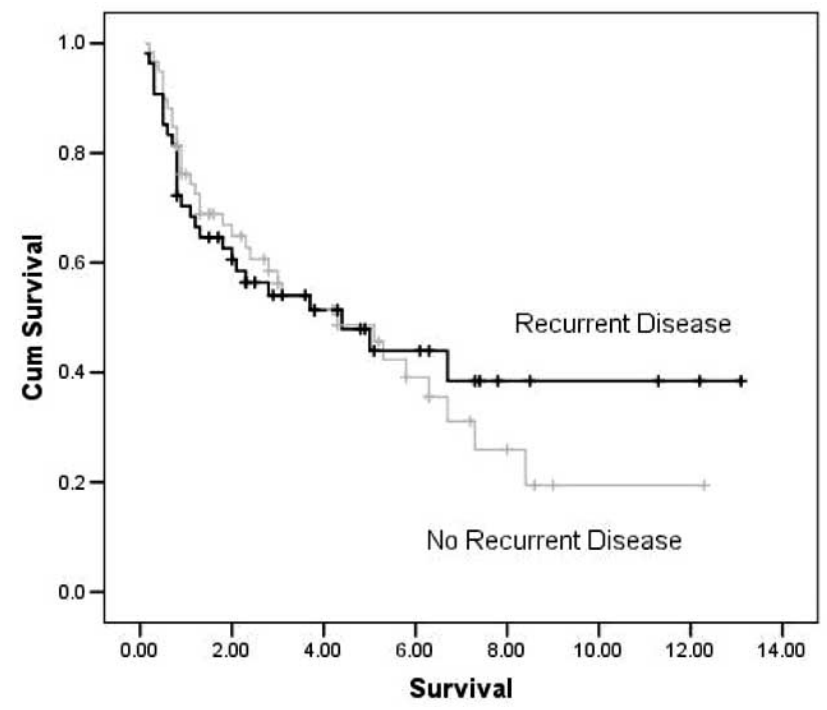

Fig. (4). Kaplan-Meier Survival Curves demonstrating differences among subgroups within the study. Graphs plot cumulative survival against survival in years. Recurrent Disease in SCC vs No Recurrent Disease in SCC, $\mathrm{p}=0.795$.

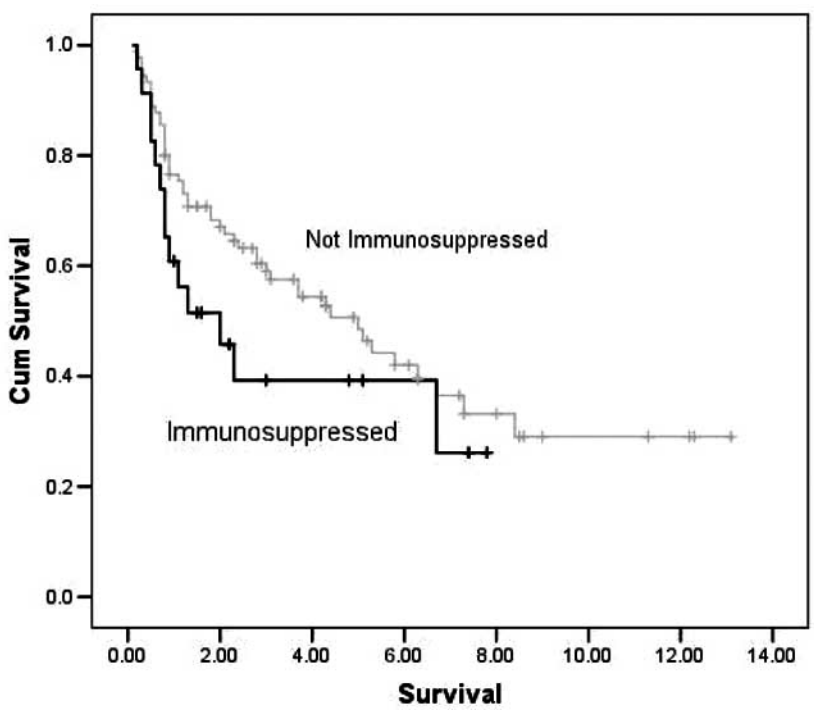

Fig. (5). Kaplan-Meier Survival Curves demonstrating differences among subgroups within the study. Graphs plot cumulative survival against survival in years. Immunosuppression in SCC vs No Immunosuppression in SCC, $\mathrm{p}=0.173$.

\section{DISCUSSION}

In this study of BCC and SCC patients seen in a head and neck surgical practice at a tertiary university medical center, the incidence of metastatic disease and recurrent disease is higher than in previously documented studies. Previous reports have quoted the incidence of metastases from cutaneous SCC between $0.5-16 \%$ [4] and BCC between $0.0028 \%$ to $0.5 \%$ [2]. The significantly higher incidence of metastatic and recurrent disease seen in our study reflects the referral pattern of our head and neck surgery practice, since patients referred to a head and neck surgeon at our institution have disease that is more likely already metastatic or recurrent.

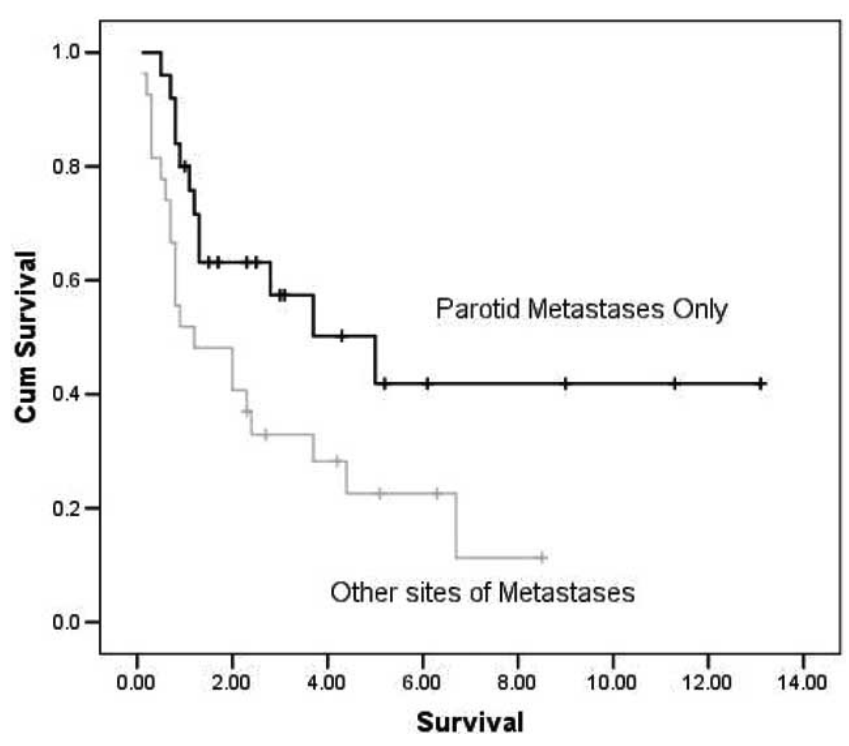

Fig. (6). Kaplan-Meier Survival Curves demonstrating differences among subgroups within the study. Graphs plot cumulative survival against survival in years. Parotid Metastases Only in SCC vs All Other Metastases in SCC, $\mathrm{p}=0.03 *$.

This highlights an important difference in the population of NMCC patients seen in our university head and neck surgical practice as compared to the populations previously described in a general dermatological setting. Poor outcomes are more likely and thus the index of suspicion for metastatic disease or recurrent disease should be higher.

The predominance of metastatic or recurrent disease also likely accounts for the ratio of SCC to BCC patients observed in this study. Previous studies have quoted a BCC:SCC ratio in the general population of 4:1 [2]. Despite BCC being four times more prevalent in the population, in our cohort, SCC was over twice as prevalent.

Squamous cell carcinoma patients in this study were more likely to have metastatic disease than BCC patients. Metastatic disease was most likely to affect the ipsilateral parotid lymph nodes first followed by spread to the cervical lymphatic chain as has been previously documented in several studies $[2,6,7]$. There was no appreciable difference between $\mathrm{BCC}$ and $\mathrm{SCC}$ patients in terms of recurrent disease. When taken together, metastases and recurrence were more likely to occur with SCC patients although the difference was not statistically significant.

In other studies, immunosuppression has been noted to increase the incidence of poor outcomes in nonmelanoma squamous cell carcinomas [5]. The minimal number of patients with immunosuppression in the BCC group did not allow for rigorous statistical analysis. $83 \%$ of immunosuppressed patients in the SCC group had poor outcomes. In reported series of SCC patients, the incidences of metastatic disease do not exceed $16 \%$ [4] and the reported incidence of recurrent disease is $28 \%$ [8]. In our study, immunosuppressed SCC patients had a higher incidence of poor outcomes $(83 \%)$ than non-immunosuppressed patients $(67 \%)$, but the difference did not reach statistical significance. Because the overall rate of poor outcomes in the SCC patients in our population was $70 \%$, it was difficult to achieve any statistically significant difference in any subgroups, including the immunosuppressed cohort. 
Reports in the literature have cited higher incidences of recurrence in immunosuppressed patients which follows intuitively [5]. Interestingly, in our cohort, there was a statistically significantly higher incidence of recurrent disease in patients without immunosuppression. This likely is a result of a longer average survival in patients without co-morbid conditions predisposing to immunosuppression. In addition, the immunosuppressed patients had a relatively higher incidence of metastatic disease which also likely decreased survival time and opportunities for developing disease recurrence.

The BCC patients in this study had a considerable survival advantage over the SCC patients. Five-year survival of the BCC group was $85 \%$ while only $50 \%$ for SCC. Based on our data, this difference in survival likely reflects the increased incidence of metastatic disease in SCC as opposed to recurrence as there was no significant difference in recurrent disease between the two groups.

Survival of SCC patients with poor outcomes was worse than patients without poor outcomes but did not reach statistical significance. At 2 years, $50 \%$ of poor outcome SCC patients survived whereas $80 \%$ of those without poor outcomes survived. By 7 years, the two populations had equivalent survival. At this junction, one can surmise that all things considered, patients were more likely to die of other illnesses than their cutaneous carcinomas. Survival of SCC patients with metastatic disease was worse than those patients without metastatic disease. However, the presence of recurrent disease alone did not portend worsened survival. The two curves overlap and interestingly, by 7 years, there was a worsened survival for patients without recurrent disease. Again, this likely reflects patients dying of other conditions than their cutaneous carcinomas.

Past literature has suggested a rate of parotid metastases of SCC at $1-3 \%$ [7]. In our study, $29 \%$ of the total cohort of SCC patients had parotid area lymph node metastases. Previous studies have suggested alterations of the cutaneous SCC staging system to differentiate and account for parotid and neck disease separately [9]. O'Brien et al. [9] suggested that patients with metastatic disease in both the parotid gland and neck have significantly worse prognosis than patients with metastatic disease in the parotid alone. In our cohort, we show that patients with parotid only disease have a survival advantage when compared to patients with neck or distant disease.

One key limitation in analyzing patients seen in a tertiary university setting is the frequent lack of appropriate follow-up and disease surveillance. After treatment of more extensive NMCC, patients frequently do not return for follow-up to a tertiary center head and neck surgeon. Often patients see the surgeon once postoperatively and are then subsequently sent back to the referring local dermatologist or primary care doctor for surveillance. Therefore, it is difficult to completely assess for metastatic disease or recurrence in the entire patient cohort. Likewise, it is thus impossible to assess for disease-specific mortality or disease-free survival without adequate postoperative visits.

In addition, for many of the patients, original pathology was unavailable for review as primary lesions had often been excised before presentation to our center for management of recurrent or metastatic disease. Lacking complete data on original pathology, we were unable to carry out detailed analysis of pathologic grade, presence of perineural invasion, or other histologic characteristics which a recent study has indicated are independent predictors of recurrence-free survival [10]. With incomplete records, multivariate analysis was not possible to be performed.

\section{CONCLUSIONS}

The incidence of poor outcomes in head and neck nonmelanoma skin cancers was higher in our series than reported in the literature. This difference may reflect the referral pattern of patients to our university otolaryngology- head and neck surgery practice. Survival patterns of our study parallel those of similar studies in the literature.

\section{ACKNOWLEDGEMENT}

The authors wish to acknowledge Alan Bostrom for his biostatistical analysis.

The authors do not have any financial or other conflicts of interest.

\section{REFERENCES}

[1] Moore BA, Weber RS, Prieto V, et al. Lymph node metastases from cutaneous squamous cell carcinoma of the head and neck. Laryngoscope 2005; 115: 1561-7.

[2] Chu A, Osguthorpe JD. Nonmelanoma cutaneous malignancy with regional metastasis. Otolaryngol Head Neck Surg 2003; 128: 66373.

[3] McGuire JF, Ge NN, Dyson S. Nonmelanoma skin cancer of the head and neck I: histopathology and clinical behavior. Am J Otolaryngol 2009; 30: 121-33.

[4] Cherpelis BS, Marcusen C, Lang PG. Prognostic factors for metastasis in squamous cell carcinoma of the skin. Dermatol Surg 2002; $28: 268-73$.

[5] Veness MJ, Porceddu S, Palme CE, et al. Cutaneous head and neck squamous cell carcinoma metastatic to parotid and cervical lymph nodes. Head Neck 2007; 29: 621-31.

[6] Ch'ng S, Maitra A, Lea R, et al. Parotid metastasis--an independent prognostic factor for head and neck cutaneous squamous cell carcinoma. J Plast Reconstr Aesthet Surg 2006; 59: 1288-93.

[7] Hinerman RW, Indelicato DJ, Amdur RJ, et al. Cutaneous squamous cell carcinoma metastatic to parotid-area lymph nodes. Laryngoscope 2008; 118: 1989-96.

[8] Veness MJ, Morgan GJ, Palme CE, et al. Surgery and adjuvant radiotherapy in patients with cutaneous head and neck squamous cell carcinoma metastatic to lymph nodes: combined treatment should be considered best practice. Laryngoscope 2005; 115: 8705 .

[9] O'Brien CJ, McNeil EB, McMahon JD, et al. Significance of clinical stage, extent of surgery, and pathologic findings in metastatic cutaneous squamous carcinoma of the parotid gland. Head Neck 2002; 24: 417-22.

[10] Kyrgidis A, Tzellos TG, Kechagias N, et al. Cutaneous squamous cell carcinoma (SCC) of the head and neck: risk factors of overall and recurrence-free survival. Eur J Cancer 2010; 46: 1563-72. 